\title{
Drivers of human gut microbial community assembly: coadaptation, determinism and stochasticity
}

\author{
Kaitlyn Oliphant ${ }^{1} \cdot$ Valeria R. Parreira $^{1} \cdot$ Kyla Cochrane $^{1} \cdot$ Emma Allen-Vercoe $^{1}{ }^{1}$
}

Received: 4 December 2018 / Revised: 21 July 2019 / Accepted: 14 August 2019 / Published online: 2 September 2019

(c) The Author(s), under exclusive licence to International Society for Microbial Ecology 2019

\begin{abstract}
Microbial community assembly is a complex process shaped by multiple factors, including habitat filtering, species assortment and stochasticity. Understanding the relative importance of these drivers would enable scientists to design strategies initiating a desired reassembly for e.g., remediating low diversity ecosystems. Here, we aimed to examine if a human fecal-derived defined microbial community cultured in bioreactors assembled deterministically or stochastically, by completing replicate experiments under two growth medium conditions characteristic of either high fiber or high protein diets. Then, we recreated this defined microbial community by matching different strains of the same species sourced from distinct human donors, in order to elucidate whether coadaptation of strains within a host influenced community dynamics. Each defined microbial ecosystem was evaluated for composition using marker gene sequencing, and for behavior using ${ }^{1} \mathrm{H}$ NMR-based metabonomics. We found that stochasticity had the largest influence on the species structure when substrate concentrations varied, whereas habitat filtering greatly impacted the metabonomic output. Evidence of coadaptation was elucidated from comparisons of the two communities; we found that the artificial community tended to exclude saccharolytic Firmicutes species and was enriched for metabolic intermediates, such as Stickland fermentation products, suggesting overall that polysaccharide utilization by Firmicutes is dependent on cooperation.
\end{abstract}

\section{Introduction}

A critical knowledge gap in the field of microbial ecology is understanding the relative contribution of the forces that drive microbial community assembly. Uncovering this information would facilitate the development of rationally designed strategies to remediate microbial communities exhibiting undesirable functionality or successive progression after a perturbation. Such forces have been proposed to include environmental selection, historical contingency, dispersal limitation and stochasticity [1]. Environmental selection additionally encompasses several distinctive subfactors that yield well to manipulation or measurement for

Supplementary information The online version of this article (https:// doi.org/10.1038/s41396-019-0498-5) contains supplementary material, which is available to authorized users.

Kaitlyn Oliphant

koliphan@uoguelph.ca

1 Department of Molecular and Cellular Biology, University of Guelph, Guelph, ON, Canada predictions, including niche availability (i.e., habitat filtering) and microbial interactivity (i.e., species assortment and coadaptation) [2-4]. Bioreactors present a promising strategy for studying the importance of such drivers, because culture conditions within them can be tightly controlled, and the use of defined microbial consortia can additionally serve to not only deconvolute the system but also to allow for manipulation to address each factor individually [5, 6]. Bioreactors are also currently utilized for both research and industrial processes [7-13], and thus observing and quantifying the contribution of these ecological forces on community assembly is in itself useful information for these applications.

The human gut microbial ecosystem (i.e., human gut microbiota) is a suitable testing ground for ecological theory. This ecosystem is known to be critical to health and well-being [14-16], with alterations in both community structure and function reported in several GI disorders $[17,18]$. Proper succession of the human gut microbiota during infancy and childhood is also essential to proper development and education of the immune system [19], with such deviations again associated with later onset of autoimmune conditions [20-22]. Therapeutics targeting the 
human gut microbiota have been trialed in such cases, including probiotics and fecal microbiota transplantation. However, the results of such clinical trials have been mixed [23-25], with several factors having been found to influence outcomes, including dosage, number of strains/donor and diet. Clearly, the availability of an ecological theoretical framework to contextualize the environmental and microbial constitution would improve the design process of these ecosystem interventions. Further, bioreactor-based models, such as the Simulator of Human Intestinal Microbial Ecosystem (SHIME) system [26, 27] and single-vessel units [13], are popular methodological approaches for investigating human gut microbial ecology, due to their replicability, sample yields, cost and lack of ethical constraints.

Thus, in our study, we aimed to quantify the relative impact of two of the drivers of microbial community assembly, environmental selection and stochasticity, in terms of both compositional species structure and metabolic behavior, through use of a defined microbial community derived from a human fecal sample and single-vessel bioreactor-based models. For the evaluation of environmental selection, we chose to utilize different medium formulations that replicate a high fiber, low protein diet and a high protein, low fiber diet, as diet has been proposed to be the dominant environmental influencer acting on the human gut microbiota [28]. We additionally scrutinized the two subfactors of environmental selection, habitat filtering and species assortment, by including a second defined microbial community matching the species constitution of the first, but where each bacterial strain was sourced from a unique human donor. Therefore, the diets would be a representation of habitat filtering, whereas the distinctive communities would model species assortment or coadaptation. To control for adaptation to the dietary condition that could occur during the initial assembly, we additionally introduced a dietary change after allowing sufficient time for community equilibration to measure the response to a relevant perturbation. Finally, for the evaluation of stochasticity, we assessed the reproducibility of replicates using several multivariate statistical methods to explore community dynamics.

\section{Materials and methods}

\section{Creation of defined microbial communities}

Two defined microbial communities were created to examine the effects of coadaptation on the dynamics of community assembly. The first community served as a control, in which all bacterial strains were derived from the same fecal sample. The second community was constructed to match the species composition of the first (as determined by aligning the $16 \mathrm{~S}$ rRNA genes from each respective pair), but with each bacterial strain sourced from a unique donor's fecal sample. The isolation methods and donor description of the control community (CC) is described in Petrof et al. [29]; however, additional species from this isolation round were added to improve the diversity of the formulation (Table S1). The same isolation techniques were utilized to derive the microbial strains for the second, "artificial" community (AC). The donors or international culture collections used to source each species of the AC are indicated in Table S1.

Genomic DNA (gDNA) isolated from each strain was individually $16 \mathrm{~S}$ rRNA gene sequenced using an Illumina MiSeq instrument (Illumina Inc., Hayward, CA, USA) in order to use the high read count output as a method to interrogate the purity of each sample. The gDNA was first extracted from each strain following the protocol described in Strauss et al. [30], and the library preparation, sequencing and data processing were then conducted as described in the $16 S$ rRNA-based compositional profiling section below. An average read depth in the $10^{3}$ range was typically achieved when sequencing single strains. The strains were decidedly pure when all amplicon sequence variants (ASVs) that could not be attributed to the target species were of low abundance $(<1 \%)$ and could be accounted for as sample cross-contamination through referencing sample blanks. Any strains that were not pure were subjected to serial dilution to extinction, as a method to improve isolation, and were then re-evaluated by the above technique.

\section{Bioreactor operation}

A $500 \mathrm{~mL}$ Multifors bioreactor system (Infors AG, Bottmingen/Basel, Switzerland) was inoculated with the defined microbial communities and operated as a model of the human distal colon as previously described [31]. Briefly, bioreactors were run under the following conditions to mimic the physiological conditions found within the human colon: (1) $37^{\circ} \mathrm{C}$, (2) $\mathrm{pH} 7.0$, (3) retention time of $24 \mathrm{~h}(500$ $\mathrm{mL}$ of feed added per vessel per day at a constant rate while maintaining volume), and (4) anaerobic conditions through sparging of $\mathrm{N}_{2}$ gas. The feed medium was designed to replicate two dietary conditions, high fiber, low protein (HF) and high protein, low fiber (HP), based upon the formulation in Marzorati et al. [32] but modified to accommodate a single-vessel system as in McDonald et al. [13] (Table S2). The study design is depicted in Fig. 1. In general, four experiments were completed, with samples taken before (B) and after (A) a dietary switch: (1) CC; HF to $\mathrm{HP}$, (2) CC; HP to HF, (3) AC; HF to HP, and (4) AC; HP to HF. Three biological replicates were taken for each experimental sampling point (every 2 days from day 2 to day 28). Technical replicates at each sampling point were 


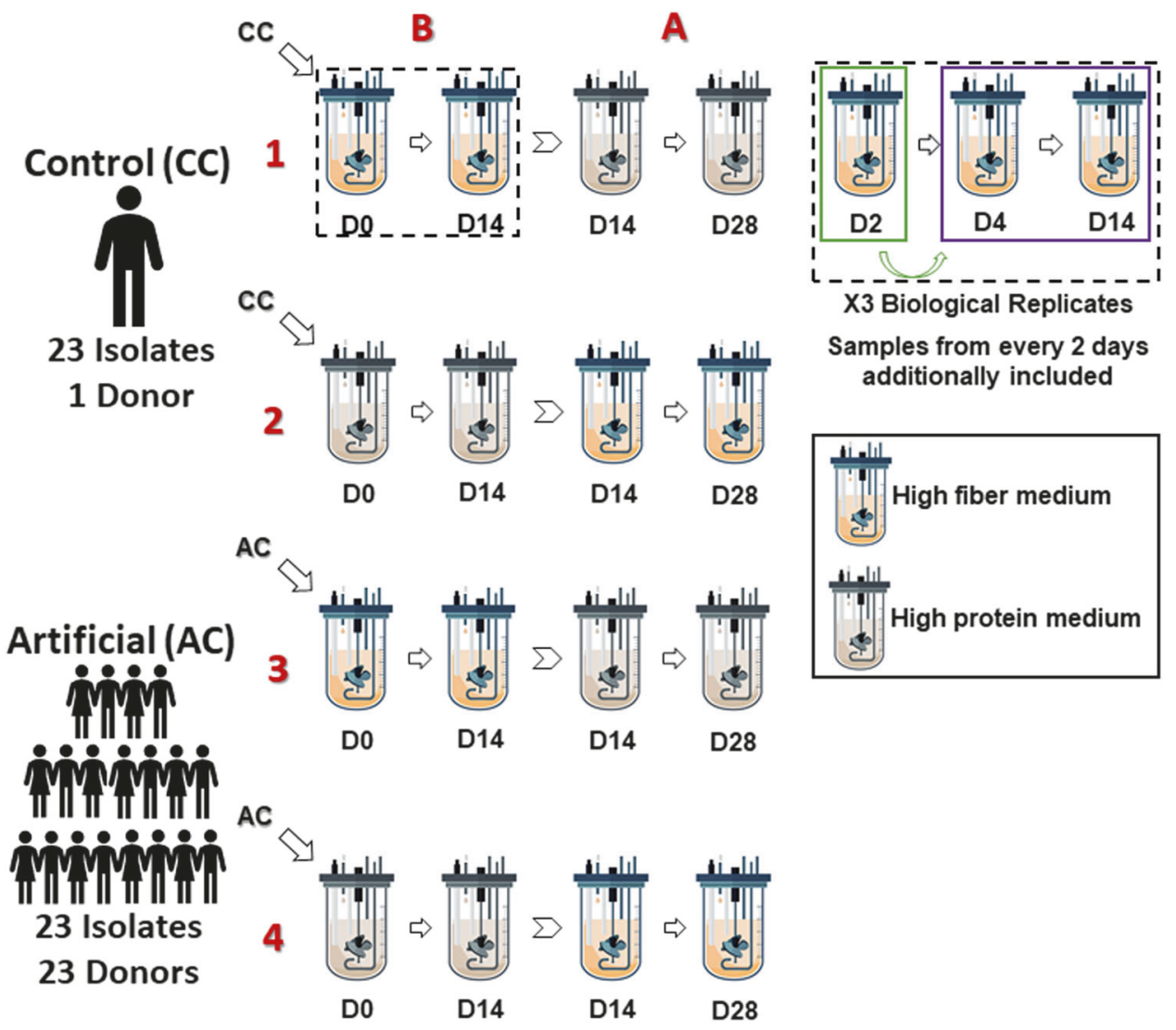

Fig. 1 Experimental design of bioreactor runs. Bioreactors were either inoculated with the control community (CC) consisting of 23 bacterial isolates from a single donor fecal sample or the artificial community (AC) consisting of 23 bacterial isolates from separate donor fecal samples that match the species of the CC by $16 \mathrm{~S}$ rRNA gene sequence similarity $(\geq 97 \%)$. Bioreactors were either fed a high fiber, low protein medium or a high protein, low fiber medium, which are distinguished by color in the figure. After allowing a 14-day equilibration (B), the medium formulations were changed, and then the bioreactors were run for an additional 14 days (A). Experiments are numbered by the

pooled for analysis, for sequencing this was done to account for amplification bias [33] and for ${ }^{1} \mathrm{H}-\mathrm{NMR}$ this was done because previous work has shown acquisition error is minimal [34].

\section{S rRNA-based compositional profiling}

The gDNA from bioreactor samples was extracted through use of the QIAamp Fast DNA Stool Mini Kit (Qiagen Inc., Germantown, MD, USA) according to the manufacturer's directions, but with extra steps included to improve cell lysis. Prior to proceeding with their recommended protocol, cells were first pelleted through centrifugation at $14,000 \mathrm{rpm}$ for $15 \mathrm{~min}$ at $4{ }^{\circ} \mathrm{C}$. After resuspension in the lysis buffer, $0.2 \mathrm{~g}$ of zirconia beads (Biospec Products Inc., Bartlesville, OK, USA) were added, then the samples were bead-beat with a Digital Disruptor Genie (Scientific microbial community and starting diet combination (1-4), with each experiment conducted in biological triplicate. Sampling occurred every 2 days, in which $2 \times 2 \mathrm{~mL}$ were taken and stored at $-80^{\circ} \mathrm{C}$ for $16 \mathrm{~S}$ rRNA gene sequencing and ${ }^{1} \mathrm{H}-\mathrm{NMR}$-based metabonomics, respectively. The box with the dotted line showcases this sampling schematic and how samples were divided into early (green box) and late (purple box) groups for moving window analysis to determine temporal stability. The comparisons conducted and statistical tests used to determine significance are outlined in Table S3

Industries Inc., New York City, NY, USA) at $3000 \mathrm{rpm}$ for $4 \mathrm{~min}$. The samples were subsequently incubated at $90{ }^{\circ} \mathrm{C}$ for $15 \mathrm{~min}$, and finally, ultrasonicated at $120 \mathrm{~V}$ for $5 \mathrm{~min}$ (Branson Ultrasonics, Danbury, CT, USA). Libraries for sequencing were constructed by a one-step PCR amplification with $400 \mathrm{ng}$ of Nextera XT Index v2 sequences (Illumina Inc.) plus standard 16S rRNA v4 region primers [35] and $2 \mu \mathrm{L}$ of gDNA template in Invitrogen Platinum PCR SuperMix High Fidelity (Life Technologies, Burlington, ON, Canada). Cycler conditions included an initial melting step of $94^{\circ} \mathrm{C}$ for $2 \mathrm{~min}$, followed by 50 cycles of $94{ }^{\circ} \mathrm{C}$ for $30 \mathrm{~s}$, annealing temperature for $30 \mathrm{~s}$ and $68^{\circ} \mathrm{C}$ for $30 \mathrm{~s}$, with a final extension step of $68^{\circ} \mathrm{C}$ for $5 \mathrm{~min}$. The annealing temperature comprised of a $0.5^{\circ} \mathrm{C}$ increment touch-down starting at $65^{\circ} \mathrm{C}$ for 30 cycles, followed by 20 cycles at $55^{\circ} \mathrm{C}$. The PCR products were subsequently purified using the Invitrogen PureLink PCR Purification Kit 
(Life Technologies) according to the manufacturer's directions. Normalization and Illumina MiSeq sequencing was carried out at the Advanced Analysis Center located in the University of Guelph, ON, Canada.

The obtained sequencing data set was processed using $\mathrm{R}$ software version 3.5 with the package DADA2 version 1.8 , following their recommended standard protocol [36]. Classification to the genus level was additionally carried out via DADA2 using the SILVA database [37] version 132. Classification to the species level, however, was conducted by uploading the ASVs to NCBI BLAST (https://blast.ncbi. nlm.nih.gov) and selecting the identification with the highest percentage and lowest e-value, while crossreferencing with the known species constitution of the defined microbial communities. The data set was then denoised by adding the ASVs that returned identical species classifications together, and after which the ASVs that equated to $<0.01 \%$ total abundance across all samples were removed. Finally, the data set was normalized by center-log ratio transformation through use of the package ALDeX2 version 1.12, taking the median of the Monte-Carlo instances as the value [38]. The statistical analysis of this data set is described in Table S3, with details provided in the supplementary information.

\section{${ }^{1}$ H-NMR-based metabonomics}

Sample preparation, ${ }^{1} \mathrm{H}-\mathrm{NMR}$ spectral acquisition and processing, and profiling of metabolites was conducted as previously described [31]. Briefly, samples were centrifuged at $14,000 \mathrm{rpm}$ (maximum speed) for $15 \mathrm{~min}$ to clarify, and the supernatants were passed through a $0.22 \mu \mathrm{m}$ filter. The addition of Chenomx internal standard (Chenomx Inc., Edmonton, AB, Canada) to the filtrates at $10 \% \mathrm{v} / \mathrm{v}$ and the scanning parameters were implemented according to the recommendations of the Chenomx NMR suite 8.3, in order to match the found compounds to their library. A Bruker Avance III $600 \mathrm{MHz}$ spectrometer with a $5 \mathrm{~mm}$ TCI 600 cryoprobe (Bruker, Billerica, MA, USA) at the Advanced Analysis Center located in the University of Guelph, ON, Canada was utilized for spectral acquisition. Spectra were collected at a sample temperature of $298 \mathrm{~K}$. The spectra were then analyzed using both an untargeted spectral binning and targeted metabolite profiling approach with the Chenomx NMR suite. For spectral binning, the default parameters of $0.04 \mathrm{ppm}$ sized bins along the 0.04-10 ppm region of the spectrum line with omission of water (4.44-5.50 ppm) and normalization by standardized area (fraction of the chemical shape indicator, DSS) were implemented. For metabolite profiling, target regions of interest in the spectra were selected by partial least squareddiscriminant analysis (PLS-DA) of the spectral binning data set (refer to supplementary information). Metabolite identifications were then based on the best fit for the peak regions with the available libraries of compounds. The libraries included both the internal set included with the Chenomx software suite, and the downloaded HMDB [39] set release 2 . The statistical analysis of both data sets is described in Table S3, with details provided in the supplementary information.

\section{Results}

\section{Determination of microbial community temporal stability and replicate reproducibility}

For this work, it was essential to first determine at which day the bioreactor-grown microbial communities had achieved a relatively stable configuration over time (i.e., temporal stability), in order to make subsequent comparisons. The $\mathrm{CC}$ reached temporal stability compositionally by day 2 and metabolically by day 4 , as determined by a lack of significant time-level variation found by both partial redundancy analysis (as a continuous variable) and repeated-measures PERMANOVA (as a categorical variable) through conducting moving window analysis, utilizing the center-log ratio transformed marker gene sequencing and ${ }^{1} \mathrm{H}-\mathrm{NMR}$ spectral binning data sets (Figs. S1, S2; Table S4). Upon dietary change from $\mathrm{HF}$ to $\mathrm{HP}$, the microbial community was compositionally similar from the first time point (day 16) until the end of the run (day 28); however, metabolic temporal stability was not reached until day 18. This latter observation is in line with the calculated amount of time it would take for the bioreactor to shed the excess $2000 \mathrm{mg} / \mathrm{mL}$ concentration of fiber from lingering fiber-rich medium following medium change to HP composition, which is 4 days post medium change (Fig. S3).

Results obtained running the AC under the same conditions were much more variable (Figs. S1, S2; Table S4). Temporal stability was reached for this community both compositionally and metabolically by day 2 in the $\mathrm{HF}$ medium, with no significant differences observed at the first time point of day 16 after the change in medium formulation. In the HP medium, however, the AC followed the patterns of the $\mathrm{CC}$ more closely than in the $\mathrm{HF}$ medium, except for taking longer to reach metabolic temporal stability ( 6 days compared to 4 days for the CC).

Next, the reproducibility between replicates of the same condition was evaluated for both the normalized sequence count and ${ }^{1} \mathrm{H}-\mathrm{NMR}$ spectral binning data sets. Statistically significant differences between replicates were found within all conditions for both data sets (Table 1). Stochastic variation was thus clearly present across each experiment, and thus in order for the changes induced by an introduced environmental pressure to be deemed statistically significant 


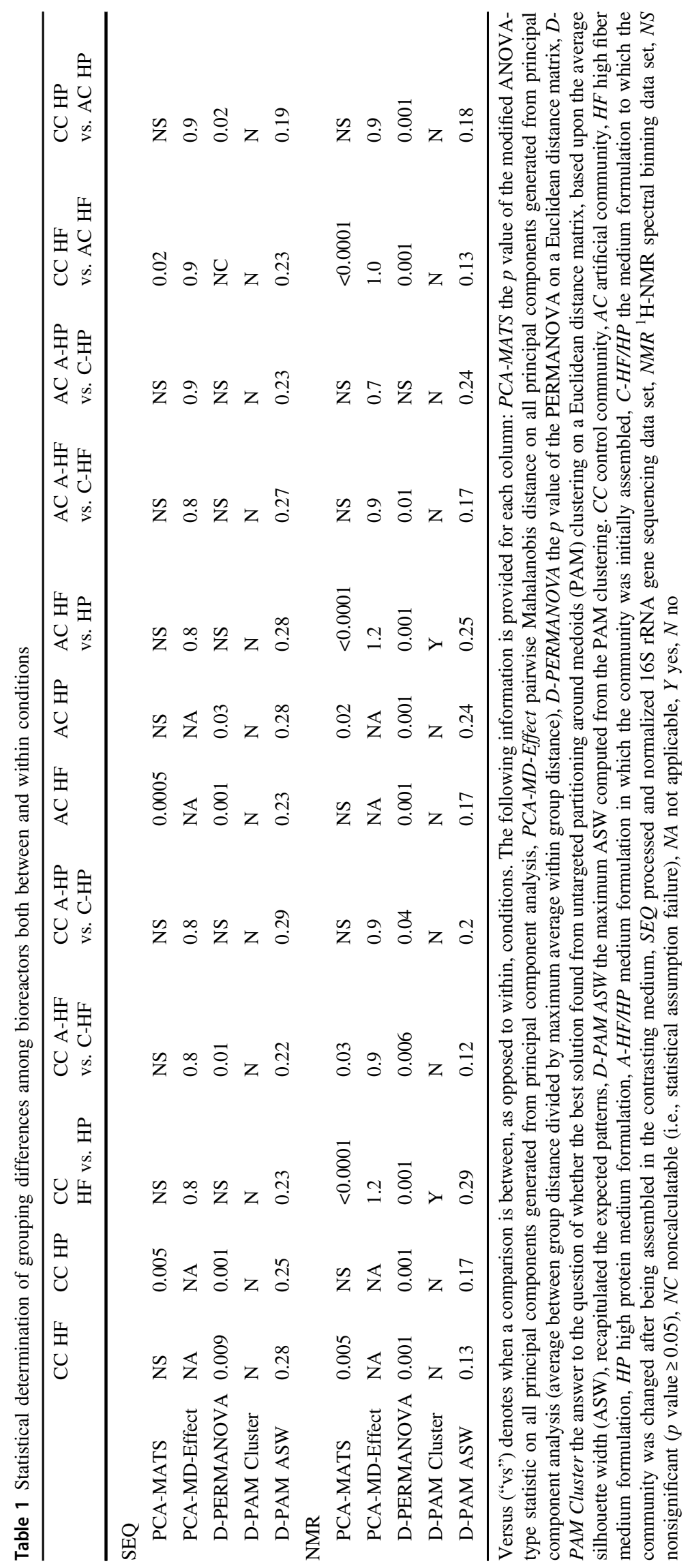


overall, we defined this to mean that it must exceed the within-condition replicate separation (Fig. 2). This could be evaluated by overlap of ellipses and magnitude of the pairwise Mahalanobis distance for the PCA approach, and recapitulation of the expected clusters by untargeted PAM clustering for the Euclidean distance approach.

The data from the $\mathrm{CC}$ collected prior to the dietary switch were used to briefly gauge the extent of difference between biological replicates (Fig. S4). The normalized sequencing data set was used to evaluate shifts in taxonomic abundance (available on public database figshare accession number: 10.6084/m9.figshare.c.4413062, with raw FASTQ files deposited in public database NCBI BioProject accession number: https://www.ncbi.nlm.nih. gov/bioproject/524005). The species that were statistically significantly different in abundance between replicates include [Eubacterium] rectale ( $q$-value $=4 \mathrm{E}-2$; effect size $=65 \%)$ and Lactobacillus paracase $i(q$ value $=4 \mathrm{E}-2$; effect size $=62 \%$ ) in the HF condition, and [Eubacterium] eligens $(q$ value $=1 \mathrm{E}-2$; effect size $=64 \%)$, Bifidobacterium pseudocatenulatum $(q$ value $=1 \mathrm{E}-2$; effect size $=58 \%)$, Escherichia coli $(q$ value $=1 \mathrm{E}-2$; effect size $=67 \%)$, Eubacterium limosum $(q$ value $=1 \mathrm{E}-2$; effect size $=66 \%), \quad$ Faecalibacterium prausnitzii $(q$ value $=1 \mathrm{E}-2$; effect size $=74 \%)$, Klebsiella aerogenes $(q$ value $=1 \mathrm{E}-2$; effect size $=78 \%$ ) and Ruminococcus faecis $(q$ value $=1 \mathrm{E}-2$; effect size $=67 \%)$ in the HP condition. Several metabolites yielded from profiling of ${ }^{1} \mathrm{H}-\mathrm{NMR}$ spectra (concentration data available on public database figshare accession number: 10.6084/m9.figshare. c.4413062) were also statistically significantly different in abundance for both conditions (Table S5), including amino acids, metabolites derived from amino acid fermentation, acetate, lactate, succinate (HP only), and uracil and alcohols (HF only).

\section{Microbial community response to dietary changes}

With the decided criteria from the above objective, i.e., the pairwise Mahalanobis distance exceeding a value of 1.0 and untargeted PAM clustering recapitulating the expected pattern of clustering samples by diet, neither community altered its composition in response to dietary change (Table 1). However, the dietary change did elicit a clear significant difference in the metabolite profile of both communities (Table 1; Fig. 2). The untargeted PAM clustering approach reliably recapitulated two clusters, each containing only the samples of one specific diet, as the best solution. The average between group Mahalanobis distance was also larger than the maximum obtained average Mahalanobis distance between replicates by a magnitude of 1.2. Finally, there was no significant difference between communities that were originally cultured in one medium compared to those eventually cultured in the same medium following a period of culture in a different medium, suggesting that within-experiment adaptation was not a confounding factor (Table 1).

Based upon the above results, we determined which features (individual taxa and metabolites) were statistically significantly different between the medium formulations of which the communities were initially grown. As expected, no statistically significant differences were found between the abundance of species (data not shown). The metabolite profiles, however, revealed $>15$ metabolites that exhibited significant changes in concentration in both the $\mathrm{CC}$ and $\mathrm{AC}$ (Table S5). The concentrations of short-chain fatty acids (SCFAs) and select metabolites of interest that were significantly different between growth medium conditions are depicted in Fig. S2 and Fig. 3 respectively. Most of these alterations were identical between each community, including increased concentrations of several amino acids and their specific fermentation by-products, a lower concentration of methanol and a higher concentration of uracil in the HP medium. Community-specific deviations included increases in concentration of several amino acids and succinate, and a decrease in concentration of valerate for the $\mathrm{CC}$ in the HP medium, whereas the AC had a higher concentration of isobutyrate and a lower concentration of glyoxylic acid.

\section{Effect of coadaptation on microbial community structure and behavior}

Finally, the differences between the CC and AC in both media were evaluated overall and between individual taxa and metabolites as above, to determine if potential coadaptation impacted community composition or behavior. With the set criteria, there were no significant differences between the overall compositional nor metabolite landscape (Table 1). However, there were several significant differences between individual taxa and metabolites. For the taxa, E. rectale $(\mathrm{HF} / \mathrm{HP} ; p$ value $=5 \mathrm{E}-6 / 4 \mathrm{E}-4$; effect size $=74 \% / 62 \%)$, Faecalicatena fissicatena $(\mathrm{HF} / \mathrm{HP} ; p$ value $=2 \mathrm{E}-5 / 6 \mathrm{E}-4$; effect size $=60 \% / 54 \%)$ and Coprococcus comes (HF only; $p$ value $=1 \mathrm{E}-5$; effect size $=65 \%$ ) were significantly altered. Upon examining the raw sequence counts, it was observed that both E. rectale and $C$. comes were virtually undetected in the $\mathrm{AC}$, with the latter likely only reaching statistical significance in the HF condition due to its relatively higher abundance in the CC. On the other hand, F. fissicatena was present in both communities, but at a lower abundance in the CC. For the metabolites, several amino acids, organic acids and uracil had increased concentrations in the $\mathrm{CC}$, whereas branchedchain fatty acids reached higher concentrations in the AC (Table S5; Fig. 3). 

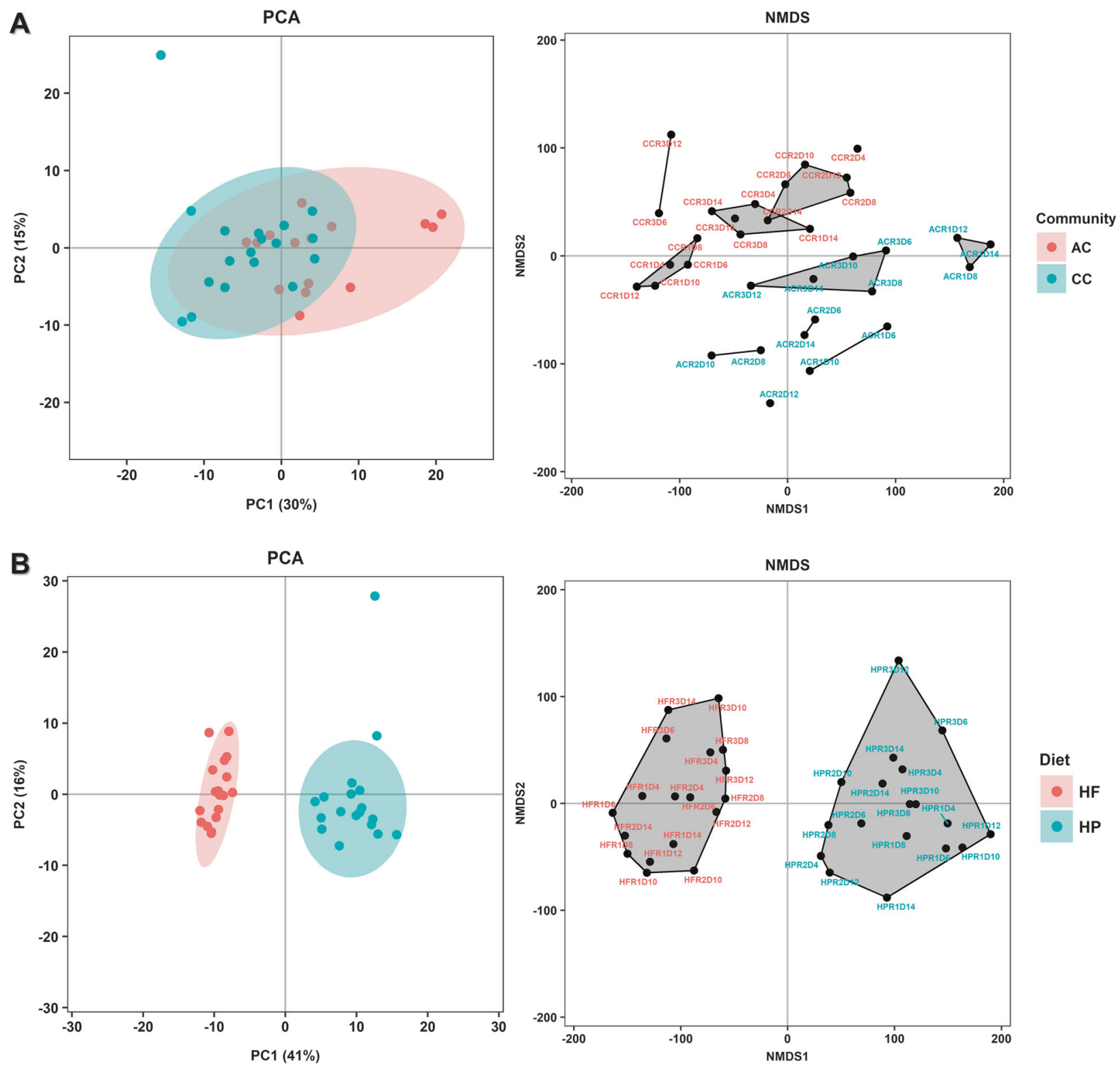

Fig. 2 Analysis of overall statistically significant differences in the ${ }^{1} \mathrm{H}$ NMR spectral binning data set obtained prior to dietary switches. Panel (a) depicts samples from days (D) 4-14 (temporally stable) of the three bioreactors fed the high protein medium formulation, grouped by microbial community. The artificial community (AC) data points are colored in pink and the control community (CC) data points are colored in blue. Samples are labeled by community, replicate (R) number (1-3) and day. Panel (b) depicts samples from days (D) 2-14 (temporally stable) of all six bioreactors grouped by medium formulation. The high fiber (HF) medium formulation data points are

colored in pink and the high protein (HP) medium formulation data points are colored in blue. Samples are labeled by medium formulation, replicate (R) number (1-3) and day. The left panel of each is the result of principal component analysis (PCA), including ellipses assuming the multivariate $t$ distribution. The right panel of each is the result of nonmultidimensional scaling (NMDS) of Euclidean distance matrices, including the best solution of untargeted partitioning around medoids clustering determined by average silhouette width. The effect of diet (panel b) exceeds stochasticity, whereas the effect of microbial community (panel a) does not

\section{Discussion}

Understanding the drivers of community succession is useful at the bench when modeling microbial ecosystems but can also translate to real-world applications. For example, for the human gut microbiota, such an understanding could assist in the design of therapeutic strategies

aiming to correct microbial derangements seen in many GI disorders, or the building of predictive tools to project changes over time (for example, during infant development). Here, we examined the relative impact of environmental selection and stochasticity on gut microbial community assembly through use of different substrate formulations. Further, we differentiated the effects of 
Fig. 3 Concentrations of select metabolites that were statistically significantly different between medium formulations or communities as determined by ${ }^{1} \mathrm{H}-\mathrm{NMR}$ metabolite profiling in bioreactor samples over time. Bioreactors $1-3$ are the communities that were initially grown in the high fiber medium formulation, whereas bioreactors 4-6 are the communities that were initially grown in the high protein medium formulation. The resultant curve from LOESS modeling is plotted for each metabolite per bioreactor. Panel (a) depicts the control community, and panel (b) depicts the artificial community.

Plots include error bars representing the expected amount of technical measurement inaccuracy $(10 \%)$
A
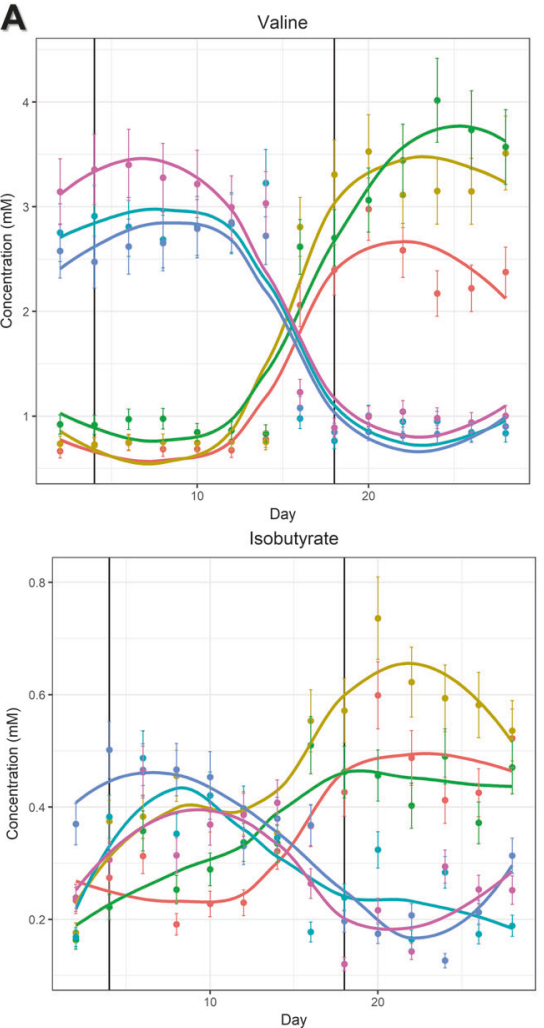

B
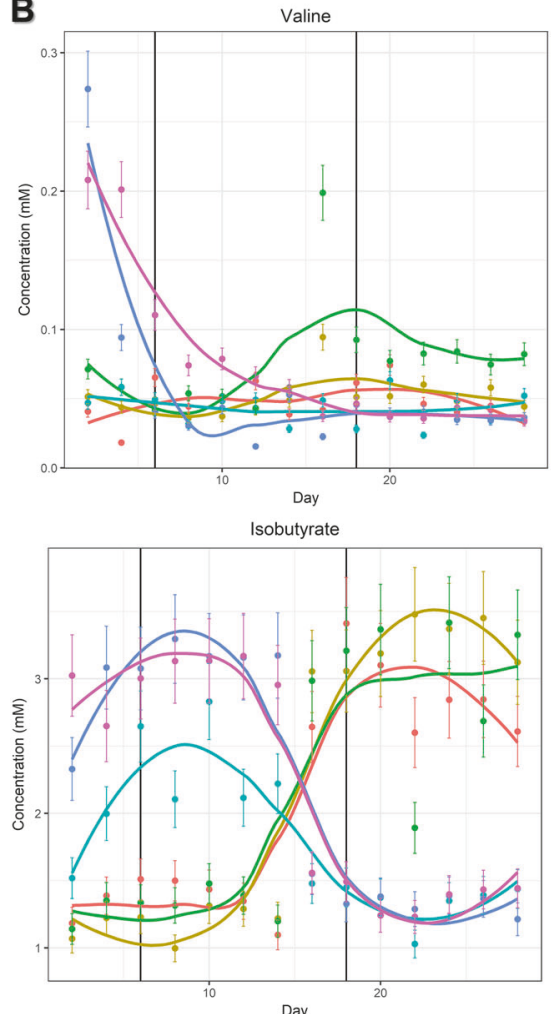

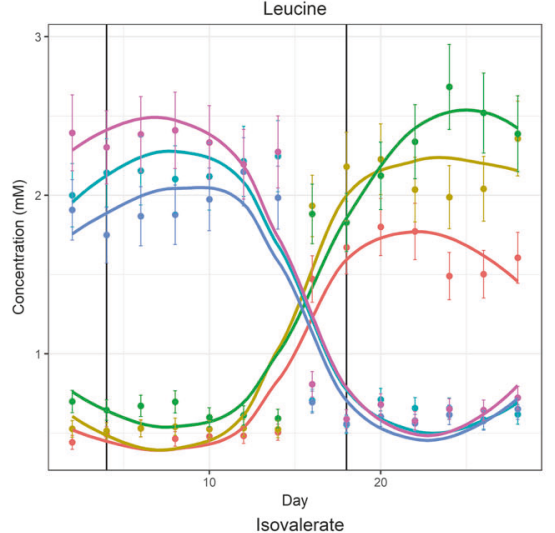

ioreactor

$\rightarrow 1$

$\rightarrow 3$

$\rightarrow 4$

$-5$

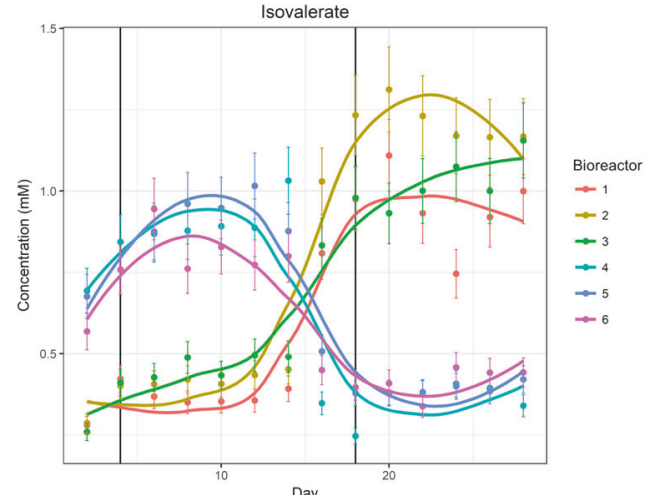

Day

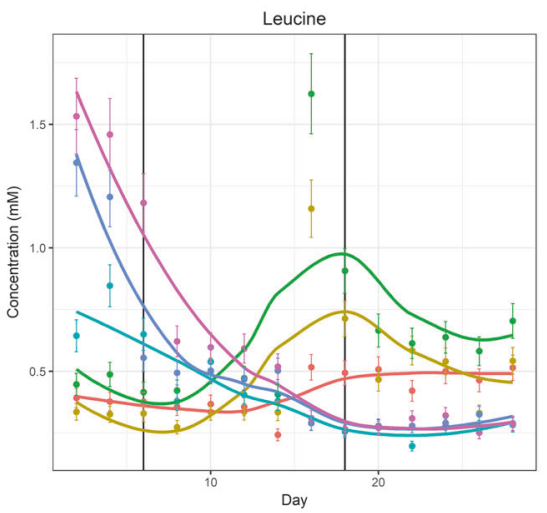

Bioreactor $\rightarrow-1$

$-3$

$-3$

$\rightarrow 5$

$\rightarrow 6$

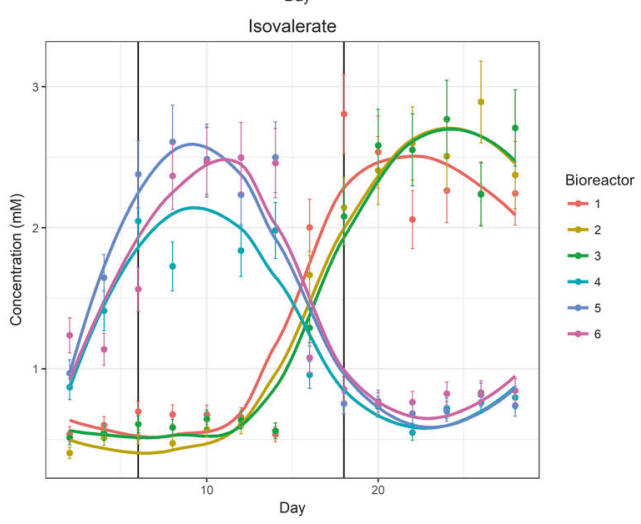

habitat and member coadaptation within environmental selection, by creating two comparative ecosystems: first, a microbial community derived from a single fecal sample, and second, an equivalent community (containing the same or highly similar species) but with each member sourced from individual donor fecal samples. 
First, it was essential to remove all forms of technical bias resulting from the use of a bioreactor-based model. It is widely reported in the literature that an initial period of adaptation occurs for microbial communities cultured in bioreactors, after which point both the composition and behavior is much more consistent throughout the experimental run [13, 26, 27]. Significant variation between replicate bioreactor runs containing the same microbial community under identical conditions has additionally previously been observed; it is unclear whether this stochasticity is a property inherent to microbial communities or is a result of batch effects [7-11]. We used a combination of redundancy analysis and a repeated-measures PERMANOVA validated by PAM clustering to determine when the adaptation period ended. In general, metabolic temporal stability was found to be attained later than compositional temporal stability in our experiments, supporting the findings of others $[26,27]$. This observation is likely to represent the shift from exponential growth phase towards finertuning of metabolic efficiency, and could be exacerbated by the relatively higher technical error introduced by marker gene sequencing when compared to ${ }^{1} \mathrm{H}-\mathrm{NMR}$ [40, 41]. The use of multivariate techniques was prudent, since we found that not all metabolites stabilized in concentration at the same time. For example, the dominant SCFAs, often steadied in concentration at a faster rate than less dominant metabolites, such as amino acid-derived fermentation byproducts (Fig. S2). We used similar multivariate techniques to determine the extent of change in microbial composition and behavior within replicates, which we recommend weighing against the differences between any applied perturbation (diets and communities in our case) to evaluate significance.

Our data suggested that growth medium significantly altered the metabolic behavior of the microbial community but not its composition. This is in line with previous observations that found only minor alterations in microbial community species structure after a dietary change [42-44] and it is also known that the composition of the human gut microbiota is robust during adulthood, with a $60 \%$ microbial strain retention rate in a 5-year window [45]. The metabolic changes we saw in both test ecosystems when diets were switched included both proteolysis (as evidenced by higher amino acid concentrations) and amino acid fermentation (as evidenced by higher concentrations of byproducts specific to these metabolisms [15, 46]), which increased in the HP medium compared to the HF medium (Fig. 3). Wu et al. found that dietary changes only influenced the species structure of the human gut microbiota when maintained for long terms [44], i.e., considerably longer than our experimental timeline of 10 days. Although it is possible that different diets may influence the total biomass of the community, and measurement of this would be of interest, we believe that microbial community adaptation is not necessary to consider as a confounding factor for the relatively short duration of our experiments.

We found that our control and artificial communities were similar to each other in terms of overall composition and metabolic behavior. However, in terms of species structure, both $E$. rectale and $C$. comes failed to integrate into the $\mathrm{AC}$, although the included strains of these species were part of the $\mathrm{CC}$. Both of these species are saccharolytic and capable of degrading fructans e.g., inulins; E. rectale can also utilize starch and xylan-derived polymers [47-49]. Intriguingly, there is evidence in the literature that these two species may share a connection; Lozupone et al. built a cooccurrence network from fecal metagenomic data collected from 124 unrelated adults and found that $C$. comes cooccurred with $E$. rectale [50]. This fits with known microbial physiology, for example, E. rectale is known to require cooperation with other species to utilize resistant starches, as it is incapable of conducting this activity on its own [51, 52]. Lozupone et al. found that Bacteroides spp. additionally co-occurred with $E$. rectale and $C$. comes, thus it is possible that the Bacteroides ovatus strains in our communities also represented potential collaborators [50]. Rakoff-Nahoum et al. elegantly showed that a strain of $B$. ovatus produced both membrane-bound and secreted forms of a glycoside hydrolase capable of degrading inulin [53]. When the secreted form of the enzyme was knocked-out, the fitness of $B$. ovatus was not impacted when grown in monoculture but was significantly diminished when grown in a community setting. Tuncil et al. additionally demonstrated that Bacteroides thetaiotaomicron and B. ovatus had reciprocal glycan substrate preferences that were maintained from monoculture to coculture [54]. These studies indicate potential mechanisms of coadaptation within a gut microbial ecosystem, such that specific species would adapt to occupy unique niches, or would collaborate to exploit the same niche (at least in terms of polysaccharide consumption). It is possible that the B. ovatus strain in the AC lacked secretory catabolic enzymes that would have assisted $E$. rectale and $C$. comes in utilizing e.g., fructans, or that the glycan substrate preference of the B. ovatus had shifted in favor of consumption of the fructans that $E$. rectale and $C$. comes could have otherwise degraded themselves, thus effectively outcompeting them and explaining their loss from the AC system over time. Alternative explanations beyond metabolism might also include a communication mismatch between strains via e.g., quorum sensing [55] or incompatible antimicrobial defense mechanisms [56]. Thus, our finding that specific strains are lost from the AC not only revealed the presence of a possible, cooperative microbial guild within the $\mathrm{CC}$, but also demonstrated that strain level variation is an important property to consider in studies aiming to examine microbial community cooperation. 
Intriguingly, we found an elevated amount of amino acid fermentation in the AC compared to the $\mathrm{CC}$, as indicated by decreased amino acid concentrations and increased concentrations of their specific fermentation by-products $[15,46]$. This heightened metabolic activity would explain the lower amount of cluster separation for the AC compared to the $\mathrm{CC}$ when considering medium formulation. We noted evidence of Stickland fermentation occurring more often in the AC compared to the $\mathrm{CC}$ (as evidenced by higher concentrations of isobutyrate, isovalerate and valerate in $\mathrm{AC}$ compared to CC samples); Stickland fermentation is a metabolism specific to the Firmicutes (usually within the Clostridia) (Fig. 3) [57, 58]. Relevant to this finding, Shoaie et al. demonstrated that when E. rectale was co-cultured with $B$. thetaiotaomicron, E. rectale switched its gene expression from a profile indicating fermentation of saccharides to one that fit with fermentation of amino acids [59]. In this case, it is likely that the E. rectale strain responded to the introduced competition by occupying a different niche. When these results are considered alongside our findings, they suggest that polysaccharide utilization by the Firmicutes is dependent on a collaborative effort between species, most likely because members of the Firmicutes tend to possess more highly specialized metabolisms [60, 61], and in the absence of such cooperation, these species will become putrefactive instead. Many GI disorders are characterized by low diversity [62], and in particular, a loss of bacterial species from clostridial cluster XIVa, including $E$. rectale and $C$. comes $[63,64]$. Further, there is often a concurrent lack of butyrate (a metabolite that is produced mainly through fermentation by certain Firmicutes members [65]), and increased inflammation (which can be promoted by the products of protein fermentation $[15,66,67])$. It is possible that an erosion of cooperative interactivity has occurred in these situations, resulting in extinction of particularly dependent species and altered behaviors from those that remain, exacerbating symptoms.

One limitation of our study was the ability to determine microbial strain purity. We introduced deep sequencing of 16S rRNA gene region (V4) amplicons obtained from each strain stock to increase the limit of detection of "contamination" by very low-level microbial species in comparison to classical streak-to-purity microbiology techniques. While this approach proved extremely helpful and gave us confidence in the purity of the majority of the isolates used for this study, there were exceptions. As the first of these, we found that Akkermansia muciniphila bloomed in the $\mathrm{CC}$ bioreactors, which was initially unexpected as it was not deliberately included in the ecosystem and not detected by our deep sequencing check. PCR was used to specifically look for Ak. muciniphila [68] using gDNA extracted from each strain stock, revealing that $A k$. muciniphila was present as a low-level contaminant of the
CC Acidaminococcus intestini stock. This result was unexpected, since an $A k$. muciniphila signal was not detected by deep 16S rRNA gene sequencing of the CC Ac. intestini stock sample ( $>10,000$ total reads) (Table S6). After carrying out a re-extraction and re-sequencing of gDNA from the Ac. intestini stock strain, this time cultured on FAA supplemented with mucin (the latter being the preferred growth substrate of Ak. muciniphila [69]), we were able to clearly detect $A k$. muciniphila, since this supplementation allowed $A k$. muciniphila to grow and become $10 \%$ of the total growth (Table S6). For the sake of our experiment, we added an appropriate, donor derived strain of Ak. muciniphila to the AC prior to conducting the bioreactor experiments for this community and then scrutinized the sequencing count data set for any other outliers once completed. Such scrutiny led to the finding of one further exception to purity: we found an unexpected number of reads classified as Phascolarctobacterium, and when Phacolarctobacterium-specific PCRs [14, 70] were conducted on the gDNA of the bioreactor samples and strains, we found Phascolarcterobacterium faecium to be present in all six replicates of the bioreactor runs, as well as the E. rectale stock, of the AC but not in the CC. We were unable to separate and recover $P$. faecium from the $E$. rectale stock culture despite multiple attempts (data not shown). Again, $P$. faecium was present at a rate of 1 per 10,000 cells of $E$. rectale. $P$. faecium did not reach high numbers in the bioreactor environment, however, as it was not statistically significantly increased in the AC when compared to the CC. We examined the possible consequences of its inclusion to our experiment. $P$. faecium is asaccharolytic and incapable of Stickland fermentation, instead consuming succinate as a substrate to produce propionate $[14,71]$. We therefore suggest that the inclusion of $P$. faecium would not have impacted upon polysaccharide utilization networks or increased putrefactive activity. One potential confounding influence $P$. faecium may have had was on succinate and propionate concentrations, as levels of succinate and propionate had decreased and increased respectively. Despite this unresolvable $P$. faecium contamination in one community but not the other, we continued with the experiment, since there is a dearth of validated alternatives for detecting contaminants present at extremely low levels, and we believe we did the best we could at ensuring strain purity given the currently available tools. However, the potential impact of $P$. faecium presence on the behavior of the AC community should not be ignored.

We conclude that stochasticity is a property inherent to human gut microbial ecosystems but is exceeded by forces of environmental selection, at least in terms of driving microbial community behavior. Substrate availability was shown to dictate functionality over cooperative interactivity, but that does not preclude the existence of coadaptation. 
Our work fits into the observations of previous studies, that indicate microbial community assembly in the human gut is a deterministic process [72], habitat filtering predominates species assortment [2], competitive interactions are more numerous than cooperative ones [73], but microbial guilds covering metabolic modules exist [3, 4]. We have also found evidence that microbial community alterations exhibited in GI disorders could result from a break-down of cooperation. Future work studying microbial interactions should consider strain level variation, and could, for example, compare interactions between strains derived from high versus low diversity ecosystems and the possibility that interactions drive the emergence of alternative stable states.

\section{Data availability}

The data sets generated during and/or analyzed during the current study are available in the figshare repository (10.6084/ m9.figshare.c.4413062), and the NCBI BioProject repository (https://www.ncbi.nlm.nih.gov/bioproject/524005).

Acknowledgements We would like to acknowledge the Natural Sciences and Engineering Research Council of Canada and Ontario Ministry of Training, Colleges and Universities scholarships to KO for providing funding. Funding was gratefully received from an NSERC Discovery Grant and a National Institutes of Health R21 (AI12157501) to EA-V. We are most thankful to our collaborators, Dr. Mike Surette, McMaster University, Canada and Dr. Sydney Finegold, VA Wadsworth Laboratory, USA for the gift of suitable matching strains for our AC community, as well as members of the EA-V lab for providing other needed isolates-Dr. Mike Toh, Michelle Daigneault, Erin Bolte and Dr. Rafael Peixoto.

\section{Compliance with ethical standards}

Conflict of interest EA-V is the co-founder and CSO of NuBiyota LLC, a company that is working to commercialize human gut-derived microbial communities for use in medical indications. The other authors declare that they have no conflict of interest.

Publisher's note: Springer Nature remains neutral with regard to jurisdictional claims in published maps and institutional affiliations.

\section{References}

1. Costello EK, Stagaman K, Dethlefsen L, Bohannan BJM, Relman DA. The application of ecological theory towards an understanding of the human microbiome. Science. 2012;336:1255-62.

2. Levy R, Borenstein E. Metabolic modeling of species interaction in the human microbiome elucidates community-level assembly rules. Proc Natl Acad Sci USA. 2013;110:12804-9.

3. Larsen OFA, Claassen E. The mechanistic link between health and gut microbiota diversity. Sci Rep. 2018;8:2183.

4. Zhang C, Yin A, Li H, Wang R, Wu G, Shen J, et al. Dietary modulation of gut microbiota contributes to alleviation of both genetic and simple obesity in children. EBioMedicine. 2015;2:968-84
5. Großkopf T, Soyer OS. Synthetic microbial communities. Curr Opin Microbiol. 2014;18:72-7.

6. Zhou J, Ning D. Stochastic community assembly: does it matter in microbial ecology? Microbiol Mol Biol Rev. 2017;81:e00002-17.

7. Pagaling E, Strathdee F, Spears BM, Cates ME, Allen RJ, Free A. Community history affects the predictability of microbial ecosystem development. ISME J. 2014;8:19-30.

8. Graham DW, Knapp CW, Van Vleck ES, Bloor K, Lane TB, Graham CE. Experimental demonstration of chaotic instability in biological nitrification. ISME J. 2007;1:385-93.

9. Zhou J, Liu W, Deng Y, Jiang Y-H, Xue K, He Z, et al. Stochastic assembly leads to alternative communities with distinct functions in a bioreactor microbial community. mBio. 2013;4:e00584-12.

10. Kohrs F, Heyer R, Bissinger T, Kottler R, Schallert K, Püttker S, et al. Proteotyping of laboratory-scale biogas plants reveals multiple steady-states in community composition. Anaerobe. 2017;46:56-68.

11. Gast CJVD, Ager D, Lilley AK. Temporal scaling of bacterial taxa is influenced by both stochastic and deterministic ecological factors. Environ Microbiol. 2008;10:1411-8.

12. Van de Wiele T, Van den Abbeele P, Ossieur W, Possemiers S, Marzorati M. The Simulator of the Human Intestinal Microbial Ecosystem (SHIME $\left.{ }^{\circledR}\right)$. In: Verhoeckx K, Cotter P, LópezExpósito I, Kleiveland C, Lea T, Mackie A, et al., editors. The impact of food bioactives on health: in vitro and ex vivo models. Cham (CH): Springer; 2015.

13. McDonald JAK, Schroeter K, Fuentes S, Heikamp-Dejong I, Khursigara CM, de Vos WM, et al. Evaluation of microbial community reproducibility, stability and composition in a human distal gut chemostat model. J Microbiol Methods. 2013;95:167-74.

14. Flint HJ, Scott KP, Duncan SH, Louis P, Forano E. Microbial degradation of complex carbohydrates in the gut. Gut Microbes. 2012;3:289-306.

15. Portune KJ, Beaumont M, Davila A-M, Tomé D, Blachier F, Sanz Y. Gut microbiota role in dietary protein metabolism and healthrelated outcomes: the two sides of the coin. Trends Food Sci Technol. 2016;57:213-32.

16. Round JL, Mazmanian SK. The gut microbiota shapes intestinal immune responses during health and disease. Nat Rev Immunol. 2009;9:313-23.

17. Cho JA, Chinnapen DJF. Targeting friend and foe: emerging therapeutics in the age of gut microbiome and disease. J Microbiol Seoul Korea. 2018;56:183-8.

18. Daliri EB-M, Tango CN, Lee BH, Oh D-H. Human microbiome restoration and safety. Int J Med Microbiol. 2018;308:487-97.

19. Renz H, Holt PG, Inouye M, Logan AC, Prescott SL, Sly PD. An exposome perspective: early-life events and immune development in a changing world. J Allergy Clin Immunol. 2017;140:24-40.

20. Fujimura KE, Sitarik AR, Havstad S, Lin DL, Levan S, Fadrosh $\mathrm{D}$, et al. Neonatal gut microbiota associates with childhood multisensitized atopy and $\mathrm{T}$ cell differentiation. Nat Med. 2016;22:1187-91.

21. Arrieta M-C, Stiemsma LT, Dimitriu PA, Thorson L, Russell S, Yurist-Doutsch S, et al. Early infancy microbial and metabolic alterations affect risk of childhood asthma. Sci Transl Med. 2015;7:307ra152.

22. Kostic AD, Gevers D, Siljander H, Vatanen T, Hyötyläinen T, Hämäläinen A-M, et al. The dynamics of the human infant gut microbiome in development and in progression toward type 1 diabetes. Cell Host Microbe. 2015;17:260-73.

23. Quraishi MN, Widlak M, Bhala N, Moore D, Price M, Sharma N, et al. Systematic review with meta-analysis: the efficacy of faecal microbiota transplantation for the treatment of recurrent and refractory Clostridium difficile infection. Aliment Pharm Ther. 2017;46:479-93. 
24. Cao Y, Zhang B, Wu Y, Wang Q, Wang J, Shen F. The value of fecal microbiota transplantation in the treatment of ulcerative colitis patients: a systematic review and meta-analysis. Gastroenterol Res Pract. 2018;2018:5480961.

25. Sun J, Marwah G, Westgarth M, Buys N, Ellwood D, Gray PH. Effects of probiotics on necrotizing enterocolitis, sepsis, intraventricular hemorrhage, mortality, length of hospital stay, and weight gain in very preterm infants: a meta-analysis. Adv Nutr. 2017;8:749-63.

26. Liu L, Firrman J, Tanes C, Bittinger K, Thomas-Gahring A, Wu GD, et al. Establishing a mucosal gut microbial community in vitro using an artificial simulator. PLoS ONE. 2018;13: e0197692.

27. Van den Abbeele P, Grootaert C, Marzorati M, Possemiers S, Verstraete W, Gérard P, et al. Microbial community development in a dynamic gut model is reproducible, colon region specific, and selective for Bacteroidetes and Clostridium cluster IX. Appl Environ Microbiol. 2010;76:5237-46.

28. $\mathrm{Xu} \mathrm{Z}$, Knight $\mathrm{R}$. Dietary effects on human gut microbiome diversity. Br J Nutr. 2015;113:S1-5.

29. Petrof EO, Gloor GB, Vanner SJ, Weese SJ, Carter D, Daigneault $\mathrm{MC}$, et al. Stool substitute transplant therapy for the eradication of Clostridium difficile infection: 'RePOOPulating' the gut. Microbiome. 2013;1:3.

30. Strauss J, White A, Ambrose C, McDonald J, Allen-Vercoe E. Phenotypic and genotypic analyses of clinical Fusobacterium nucleatum and Fusobacterium periodonticum isolates from the human gut. Anaerobe. 2008;14:301-9.

31. Yen S, McDonald JAK, Schroeter K, Oliphant K, Sokolenko S, Blondeel EJM, et al. Metabolomic analysis of human fecal microbiota: a comparison of feces-derived communities and defined mixed communities. J Proteome Res. 2015;14:1472-82.

32. Marzorati M, Vilchez-Vargas R, Bussche JV, Truchado P, Jauregui R, El Hage RA, et al. High-fiber and high-protein diets shape different gut microbial communities, which ecologically behave similarly under stress conditions, as shown in a gastrointestinal simulator. Mol Nutr Food Res. 2017;61.

33. Kennedy K, Hall MW, Lynch MDJ, Moreno-Hagelsieb G, Neufeld JD. Evaluating bias of illumina-based bacterial 16S rRNA gene profiles. Appl Env Microbiol. 2014;80:5717-22.

34. Schreier C, Kremer W, Huber F, Neumann S, Pagel P, Lienemann $\mathrm{K}$, et al. Reproducibility of NMR analysis of urine samples: impact of sample preparation, storage conditions, and animal health status. BioMed Res Int. https://www.hindawi.com/journals/ bmri/2013/878374/. Accessed 12 Feb 2019.

35. Caporaso JG, Lauber CL, Walters WA, Berg-Lyons D, Lozupone CA, Turnbaugh PJ, et al. Global patterns of $16 \mathrm{~S}$ rRNA diversity at a depth of millions of sequences per sample. Proc Natl Acad Sci USA. 2011;108(Suppl 1):4516-22.

36. Callahan BJ, McMurdie PJ, Rosen MJ, Han AW, Johnson AJA, Holmes SP. DADA2: High-resolution sample inference from Illumina amplicon data. Nat Methods. 2016;13:581-3.

37. Quast C, Pruesse E, Yilmaz P, Gerken J, Schweer T, Yarza P, et al. The SILVA ribosomal RNA gene database project: improved data processing and web-based tools. Nucleic Acids Res. 2013;41:D590-6.

38. Fernandes AD, Reid JN, Macklaim JM, McMurrough TA, Edgell DR, Gloor GB. Unifying the analysis of high-throughput sequencing datasets: characterizing RNA-seq, 16S rRNA gene sequencing and selective growth experiments by compositional data analysis. Microbiome. 2014;2:15.

39. Wishart DS, Tzur D, Knox C, Eisner R, Guo AC, Young N, et al. HMDB: the Human Metabolome Database. Nucleic Acids Res. 2007;35:D521-6

40. Sokolenko S, Blondeel EJM, Azlah N, George B, Schulze S, Chang $\mathrm{D}$, et al. Profiling convoluted single-dimension proton
NMR spectra: a Plackett-Burman approach for assessing quantification error of metabolites in complex mixtures with application to cell culture. Anal Chem. 2014;86:3330-7.

41. Bender JM, Li F, Adisetiyo H, Lee D, Zabih S, Hung L, et al. Quantification of variation and the impact of biomass in targeted 16S rRNA gene sequencing studies. Microbiome. 2018;6:155.

42. Duncan SH, Belenguer A, Holtrop G, Johnstone AM, Flint HJ, Lobley GE. Reduced dietary intake of carbohydrates by obese subjects results in decreased concentrations of butyrate and butyrate-producing bacteria in feces. Appl Environ Microbiol. 2007;73:1073-8

43. Walker AW, Ince J, Duncan SH, Webster LM, Holtrop G, Ze X, et al. Dominant and diet-responsive groups of bacteria within the human colonic microbiota. ISME J. 2011;5:220-30.

44. Wu GD, Chen J, Hoffmann C, Bittinger K, Chen Y-Y, Keilbaugh $\mathrm{SA}$, et al. Linking long-term dietary patterns with gut microbial enterotypes. Science. 2011;334:105-8.

45. Faith JJ, Guruge JL, Charbonneau M, Subramanian S, Seedorf H, Goodman AL, et al. The long-term stability of the human gut microbiota. Science. 2013;341:1237439.

46. Kanehisa M, Goto S. KEGG: Kyoto Encyclopedia of Genes and Genomes. Nucleic Acids Res. 2000;28:27-30.

47. Scott KP, Martin JC, Duncan SH, Flint HJ. Prebiotic stimulation of human colonic butyrate-producing bacteria and bifidobacteria, in vitro. FEMS Microbiol Ecol. 2014;87:30-40.

48. Rosero JA, Killer J, Sechovcová H, Mrázek J, Benada O, Fliegerová K, et al. Reclassification of Eubacterium rectale (Hauduroy et al. 1937) Prévot 1938 in a new genus Agathobacter gen. nov. as Agathobacter rectalis comb. nov., and description of Agathobacter ruminis sp. nov., isolated from the rumen contents of sheep and cows. Int J Syst Evol Microbiol. 2016;66:768-73.

49. Holdeman LV, Moore WEC. Coprococcus, twelve new species, and emended descriptions of four previously described species of bacteria from human feces. Int $J$ Syst Evol Microbiol 1974;24:260-77.

50. Lozupone C, Faust K, Raes J, Faith JJ, Frank DN, Zaneveld J, et al. Identifying genomic and metabolic features that can underlie early successional and opportunistic lifestyles of human gut symbionts. Genome Res. 2012;22:1974-84.

51. Cockburn DW, Orlovsky NI, Foley MH, Kwiatkowski KJ, Bahr $\mathrm{CM}$, Maynard M, et al. Molecular details of a starch utilization pathway in the human gut symbiont Eubacterium rectale. Mol Microbiol. 2015;95:209-30.

52. Cockburn DW, Suh C, Medina KP, Duvall RM, Wawrzak Z, Henrissat B, et al. Novel carbohydrate binding modules in the surface anchored $\alpha$-amylase of Eubacterium rectale provide a molecular rationale for the range of starches used by this organism in the human gut. Mol Microbiol. 2018;107:249-64.

53. Rakoff-Nahoum S, Foster KR, Comstock LE. The evolution of cooperation within the gut microbiota. Nature. 2016;533:255-9.

54. Tuncil YE, Xiao Y, Porter NT, Reuhs BL, Martens EC, Hamaker BR. Reciprocal prioritization to dietary gycans by gut bacteria in a competitive environment promotes stable coexistence. mBio. 2017;8:e01068-17.

55. Kerényi Á, Bihary D, Venturi V, Pongor S. Stability of multispecies bacterial communities: signaling networks may stabilize microbiomes. PLoS ONE. 2013;8:e57947.

56. Cordero OX, Wildschutte H, Kirkup B, Proehl S, Ngo L, Hussain $\mathrm{F}$, et al. Ecological populations of bacteria act as socially cohesive units of antibiotic production and resistance. Science. 2012;337:1228-31.

57. Fischbach MA, Sonnenburg JL. Eating for two: how metabolism establishes interspecies interactions in the gut. Cell Host Microbe. 2011;10:336-47.

58. de Vladar HP. Amino acid fermentation at the origin of the genetic code. Biol Direct. 2012;7:6. 
59. Shoaie S, Karlsson F, Mardinoglu A, Nookaew I, Bordel S, Nielsen J. Understanding the interactions between bacteria in the human gut through metabolic modeling. Sci Rep. 2013;3:2532.

60. Rogowski A, Briggs JA, Mortimer JC, Tryfona T, Terrapon N, Lowe EC, et al. Glycan complexity dictates microbial resource allocation in the large intestine. Nat Commun. 2015;6:7481.

61. Cho I, Blaser MJ. The Human Microbiome: at the interface of health and disease. Nat Rev Genet. 2012;13:260-70.

62. Lane ER, Zisman TL, Suskind DL. The microbiota in inflammatory bowel disease: current and therapeutic insights. J Inflamm Res. 2017;10:63-73.

63. Vital M, Karch A, Pieper DH. Colonic butyrate-producing communities in humans: an overview using omics data. mSystems. 2017;2: 00130-17.

64. Louis P, Flint HJ. Diversity, metabolism and microbial ecology of butyrate-producing bacteria from the human large intestine. FEMS Microbiol Lett. 2009;294:1-8.

65. Fan P, Li L, Rezaei A, Eslamfam S, Che D, Ma X. Metabolites of dietary protein and peptides by intestinal microbes and their impacts on gut. Curr Protein Pept Sci. 2015;16:646-54.

66. Yao CK, Muir JG, Gibson PR. Review article: insights into colonic protein fermentation, its modulation and potential health implications. Aliment Pharm Ther. 2016;43:181-96.
67. Collado MC, Derrien M, Isolauri E, de Vos WM, Salminen S. Intestinal integrity and Akkermansia muciniphila, a mucindegrading member of the intestinal microbiota present in infants, adults, and the elderly. Appl Environ Microbiol. 2007;73:7767-70.

68. Derrien M, Vaughan EE, Plugge CM, de Vos WM. Akkermansia muciniphila gen. nov., sp. nov., a human intestinal mucindegrading bacterium. Int J Syst Evol Microbiol. 2004;54:1469-76.

69. Wu F, Guo X, Zhang J, Zhang M, Ou Z, Peng Y. Phascolarctobacterium faecium abundant colonization in human gastrointestinal tract. Exp Ther Med. 2017;14:3122-6.

70. Watanabe Y, Nagai F, Morotomi M. Characterization of Phascolarctobacterium succinatutens sp. nov., an Asaccharolytic, succinate-utilizing bacterium isolated from human feces. Appl Env Microbiol. 2012;78:511-8.

71. Del Dot T, Osawa R, Stackebrandt E. Phascolarctobacterium faecium gen. nov, spec. nov., a novel taxon of the Sporomusa group of bacteria. Syst Appl Microbiol. 1993;16:380-4.

72. Jeraldo P, Sipos M, Chia N, Brulc JM, Dhillon AS, Konkel ME, et al. Quantification of the relative roles of niche and neutral processes in structuring gastrointestinal microbiomes. Proc Natl Acad Sci USA. 2012;109:9692-8.

73. Coyte KZ, Schluter J, Foster KR. The ecology of the microbiome: networks, competition, and stability. Science. 2015;350:663-6. 